\title{
Non-Adiabatic Motion of Charged Particles Traversing a Weak Magnetic Field: Pitch Angle Scattering
}

\author{
By Sydney Chapman'1) and Peter C. Kendall ${ }^{2}$ )
}

\begin{abstract}
Summary - A charged particle moves with velocity $\boldsymbol{v}$ in a constant non-uniform magnetic field $\boldsymbol{H}$, spiralling with Larmor radius $R$. If $R$ is small compared with the scale length $L$ of the field, the magnetic moment associated with the Larmor motion of the particle is nearly constant. Consequently $\theta$, the ('pitch') angle between $\boldsymbol{v}$ and $\boldsymbol{H}$, varies as arcsin $H^{1 / 2}$. Hence $\theta$ in such adiabatic motion is approximately the same at points on the path where $H$ has the same value. But the magnetic moment and the pitch angle may differ materially at two such points, each in the region where $R / L$ is small, if between them the particle traverses a region where $R / L$ is not small. This region of non-adiabatic motion 'scatters' the pitch angles.

Such scattering is investigated for regions of weak field ( $R$ large), with and without the presence of a neutral line along which $H=0$. Either type of region, it is found, can scatter the pitch angles. This gives support to the theory proposed by AKASOFU and CHAPMAN to explain why auroral arcs and bands are very thin.

The scattering here examined is of interest also in connection with magnetic mirror devices for nuclear energy transformation. It may also have applications to phenomena of solar and stellar atmospheres.
\end{abstract}

\section{Introduction}

A particle of mass $m$ and charge $e$ (in e.m. units) moving with velocity $\boldsymbol{v}$ in a constant magnetic field $\boldsymbol{H}$ maintains a constant speed $\boldsymbol{v}$. If the field is uniform, the path is a helix with mean motion in the direction $\pm \boldsymbol{H}$, with a ('Larmor') radius $R$ given by $m v \sin \theta /|e| H$, where $\theta$ denotes the ('pitch') angle between $\boldsymbol{v}$ and $\boldsymbol{H}$; a magnetic moment $\mu$, given by $\left[\left(m v^{2} / 2\right) / H\right] \sin ^{2} \theta$, is associated with the circular part of the motion. If the field is non-uniform, with scale length $L$, the particle spirals in approximately Larmorian manner, and $\mu$ is almost constant, provided that $R / L$ is small $\left.[1]^{3}\right)$, the 'guiding' or Alfvén centre of the circular part of the motion moves nearly along a field line, though with a slight drift transverse to the lines. Hence $\sin \theta$ varies along the path approximately as $H^{1 / 2}$, and $\theta$ has nearly equal values at two points $P, P^{\prime}$ if $H$ has the same value at each. If $H_{0}$ is the minimum value of $H$ along the path of the particle, and if $\theta_{0}$ is the pitch angle there (its minimum value), then $\mu=\left[\left(m v^{2} / 2\right) / H_{0}\right]$ $\sin ^{2} \theta_{0}$. The particle moves nearly along the field line in one direction until it reaches

1) Geophysical Institute, College, Alaska; High Altitude Observatory, Boulder, Colorado (there engaged in a program of research sponsored jointly by the National Bureau of Standards and the Air Force Geophysical Directorate); and Institute of Science and Technology, University of Michigan, Ann Arbor, Michigan.

2) High Altitude Observatory, Boulder, Colorado and Department of Applied Mathematics, University of Sheffield.

3) Numbers in brackets refer to References, page 122. 
a point at which $\sin \theta=1$; there $H=\left(m v^{2} / 2\right) / \mu=H_{0} / \sin ^{2} \theta_{0}$; at such a ('mirror') point the motion along the line is reduced to zero, and the particle then returns nearly along the field line in the reverse direction.

The aurora polaris is caused by the entry of high speed electrons and protons into the atmosphere. They spiral downwards along the geomagnetic field lines, which in the usual auroral latitudes are nearly vertical. Overcoming atmospheric retardation they descend to about $100 \mathrm{~km}$ above the earth's surface. Extrapolating back along the field lines, from the latitudes of entry, one can estimate the distance $f a$ ( $a=$ the earth's radius) at which they cross the (magnetic) equatorial plane. In so doing, to a first approximation the field lines may be taken as those of the dipole component of the field; a closer approximation is obtained by taking account of the non-dipole parts of the field; but allowance should also be made for possible distortion of the lines by electric currents in the region of the Van Allen belts $[2,3]$. It seems that during ordinary auroral occurrences $f$ is about 6 ; during great magnetic storms it may decrease to around 3 . The normal minimum value of $H$ along the path is approximately $0.3 / f^{3}$ gauss. In the auroral zone $H$ is about 0.6 gauss. Particles that can reach to this field strength from $f=6$ must cross the equatorial plane with a very small pitch angle, less than $3^{\circ}$.

Such particles, entering the atmosphere, do not return therefrom; they are lost from the belts. All such particles with very small pitch angles would disappear from the belts in a few seconds unless the supply were maintained in some way. Quiet auroras at times continue in the same position for minutes on end. This implies a continuing supply of particles of small pitch angle. Akasofu and CHAPMAN [4] linked this supply with pitch-angle scattering, which they considered would occur in a long laterally restricted region of low field strength in or near the equatorial plane at the distance $f a$. The supply would be drawn from the main distribution of particles, with pitch angles too large, at or near the equatorial plane, to enable them to enter the atmosphere, were it not that in traversing the said region their pitch angles are reduced.

The present study of pitch angle scattering was undertaken in order to check their supposition that a region of weak equatorial field would be an effective scatterer of pitch angles. They proposed that the scattering region is associated with a neutral line in the field, where $H=0$; they took this line to be one border of a thin strip in the equatorial plane, within which the ring current reverses the normal field direction. It is not certain that such reversal does or can occur. Gold [5] and PARKER [6] suggested that the postulated scattering might occur in a region of reduced field strength without a reversal (CHAPMAN [7]). Hence we have considered both types of weak field, with and without a neutral line. We find that either can produce the required scattering.

Our results also bear on the motion of charged particles in the magnetic mirror devices used in studies of nuclear energy transformation.

In general the paths cannot be expressed in any convenient analytical form; hence our investigation is necessarily a numerical one.

We have, however, been able to treat analytically [8] an analogous problem of scattering, in a type of field in which the particle motion is non-adiabatic and $R / L$ is large, not because of the weakness of the field, but because $L$ becomes small, indeed zero, at certain field discontimities. 
In both investigations our main interest is in the production of small pitch angles. As the motion is reversible, it is convenient and suitable to consider paths with small initial pitch angle, and to study how the pitch angle and magnetic moment are changed by traversing the scattering region.

The present study may be called topological as opposed to statistical; it is concerned with the nature and space distribution of paths of particles that after traversing the region where $R / L$ is large have small pitch angles. A statistical investigation is desirable that would give the rate of supply of particles of small pitch angle in terms of the space and velocity distribution of the particles incident on the region of large $R / L$. Such an investigation would be much more extensive than the one here described, and we have not yet attempted it.

\section{Pitch angle scattering by field discontimuities}

In our earlier paper [8] we considered the field indicated in Figure 1, associated with orthogonal right-handed Cartesian coordinates $x, y, z$ measured along the directions of a triad $\boldsymbol{i}, \boldsymbol{j}, \boldsymbol{k}$ of unit vectors. The field strength $H$ is everywhere the same, and $H$ is uniform in each of the four regions marked as $Y, Z,-Y,-Z$ in Figure 1, but the directions are different, being respectively along $-\boldsymbol{j}, \boldsymbol{k}, \boldsymbol{j}$ and $-\boldsymbol{k}$. Thus the
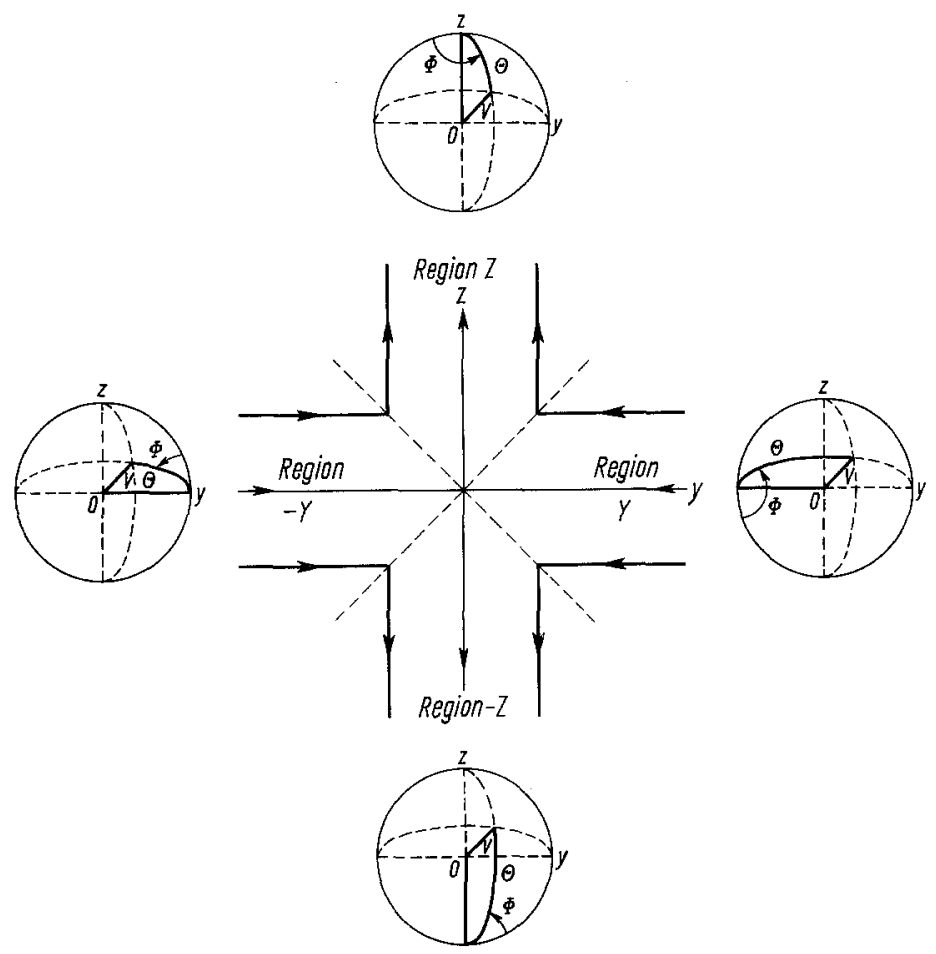

Figure 1

A discontinuous field model of the magnetic intensity near a straight neutral line, together with the definition of azimuthal angle in the four regions 
field is discontinuous at the planes $y= \pm z$. In each region the Larmor radius, the magnetic moment and the pitch angle of a moving charged particle are all strictly constant; but they may change discontinuously on crossing from one region to another. The speed $v$, however, is constant throughout.

It is convenient to take units $L_{0}$ and $T_{0}$ of length and time such that the speed, and the Larmor radius for transverse motion with this speed, are each unity. In terms of the measures initially used,

$$
L_{0}=\frac{m v}{|e| H},
$$

where the charge $e$ of the particle is expressed in e.m.u; and

$$
T_{0}=\frac{m}{|e| I I} .
$$

In terms of these units, the Larmor radius for a particle with pitch angle $\theta$ is $\sin \theta$, and the magnetic moment is given by

$$
\mu=\mu_{0} \sin ^{2} \theta, \quad \mu_{0}=\frac{m v^{2}}{2 H}=\frac{m}{2 H} .
$$

In each region the mean motion $\boldsymbol{w}$ of a particle is along the field direction, in one or other sense, so that $\boldsymbol{w}= \pm \boldsymbol{j} \cos \theta$ in the regions $\mathrm{Y}$ and $-\mathrm{Y}$, and $\boldsymbol{w}= \pm \boldsymbol{k} \cos \theta$ in the regions $Z$ and $-Z$.

\section{2 a Particles with zero initial pitch angle}

All types of phenomena are illustrated by considering particles initially moving leftward in the upper half $(z>0)$ of region $\mathrm{Y}$ with $z$-coordinate $z_{0}$. We found that if the pitch angle in $\mathrm{Y}$ is zero, the paths are of three distinct types, for three ranges of $z_{0}$; $\mathrm{N}$ (near), I (intermediate) and $\mathrm{F}$ (far). They may be classified thus:

$\begin{array}{lllc} & & \text { Region of exit } \\ z_{0} \text { range } \mathrm{N}, & \text { continuing orbits; } & \text { for } 0<z_{0}<0.474 & -\mathrm{Y} \\ z_{0} \text { range } \mathrm{I}, & \text { deflected orbits; } & \text { for } 0.474<z_{0}<0.5 & -\mathrm{Z} \\ z_{0} \text { range } \mathrm{F}, & \text { returned orbits; } & \text { for } z_{0}>0.5 & \mathrm{Y}\end{array}$

The simplest orbit is that for $z_{0}=0$; in this case the particle moves uniformly along the $y$-axis, passing from $Y$ to $-Y$ through the $x$-axis. For $z_{0}>0$, on crossing from $Y$ to $Z$, the pitch angle suddenly changes from 0 to $90^{\circ}$, and in region $Z$ the path is a circular arc of unit radius in the plane $z=z_{0}$. If $z_{0}>0.5$ the particle returns to $\mathrm{Y}$ after describing a semicircular arc; then it moves with reversed motion to infinity along a line displaced by two units of length from its original path. The displacement is in the direction $(e\|e\|) \boldsymbol{k} \times \boldsymbol{j}$, that is $-(e \| e \mid) \boldsymbol{i}$, opposite for charges of different signs.

If, however, $z_{0}<0.5$ the path in $Z$ meets the other boundary of the region, the plane $y=-z$, in the plane $z=z_{0}$. There it enters the region $-\mathrm{Y}$, with a pitch angle that depends on $z_{0}$. It is $90^{\circ}$ for $z_{0}=0.5$, and less for smaller values of $z_{0}$. 
Our calculations showed that if $z_{0}>0.474$ the helical path in $-\mathrm{Y}$ will meet the other boundary of $-\mathrm{Y}$, where the particle will cross into the region $-Z$. Figure 2 shows the limiting path of this type for $z_{0}=0.5$; the pitch angle is $90^{\circ}$ in $Z$ and in $-\mathrm{Y}$, and zero in $-Z$. For $z_{0}<0.5$ the pitch angle in $-\mathrm{Y}$ is $\arcsin \left(2 z_{0}\right)$. If $z_{0}<0.474$ the helical path in $-Y$ does not meet the boundary of -- Z. In that case the final mean motion of the particle is along the original direction, $-\boldsymbol{j}$, but the path is changed by the passage of the particle through $Z$ from being a straight line to a helix, with displacement of the helix axis from the original path, in both the $\boldsymbol{i}$ and $\boldsymbol{k}$ directions. For $z_{0}=0.474$, the helix touches the boundary between $-\mathrm{Y}$ and $-Z$, and the path may continue in either region; in $-Z$ the pitch angle will be less than $90^{\circ}$. The paths for $z_{0}$ range $I$ enter $-Z$, and the mean motion is deflected though $90^{\circ}$.

It is convenient to represent $v$ [expressed in the units $(2.1),(2.2)$, so that $v=1$ ] by a velocity point $Q$ on a sphere of unit radius in an auxiliary 'velocity' space. For the paths with zero initial pitch angle here considered, the initial position $\left(Q_{0}\right)$ of $Q$ has the position vector - $j$ relative to the centre of the sphere. After the particle has crossed into $Z, Q$ moves uniformly from $Q_{0}$ along the great circle in the plane normal to $\boldsymbol{k}$. If the particle moves into $-Y$, when $Q$ has the position $Q^{\prime}$ on this circle, the subsequent path of $Q$ is along a small circle with pole $Q_{0}$. If the particle later moves into $-Z$, when $Q$ has the position $Q^{\prime \prime}$, the further locus of $Q$ is a small circle with its pole at $\boldsymbol{k}$ or $-\boldsymbol{k}$ on the unit sphere.

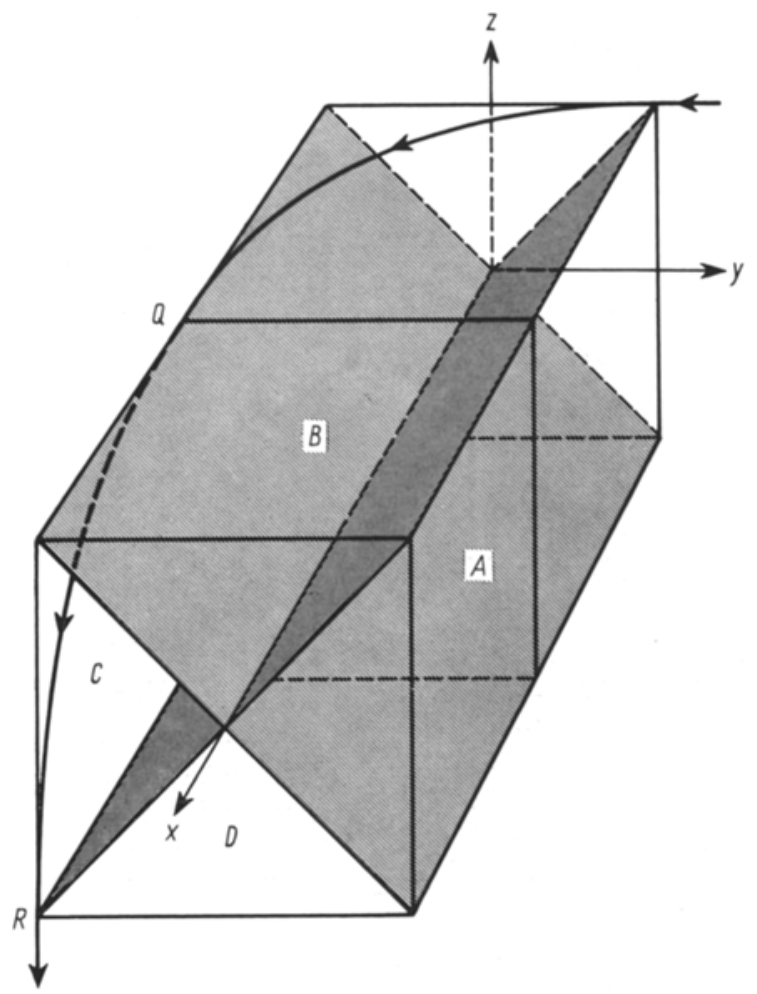

Figure 2

The limiting path for $z_{0}=0.5$ in the model field of Figure 1 


\section{$2 b$ Particles with small initial pitch angle $\theta_{0}$}

When the particle moves in $\mathrm{Y}$ with a small pitch angle $\theta$ (in circular measure), different from zero, the direction of $\boldsymbol{v}$ involves an azimuthal angle $\phi$ as well as the magnitude $\theta$. This azimuth is measured by the angle between two half planes each bordered by the tangent to the field line through $P$, the point occupied by the particle at the instant. One of the half planes contains $\boldsymbol{v}$, the other may be chosen at will. Figure 1 shows suitable choices for the four regions. To the first order in $\theta, \boldsymbol{v}=-\boldsymbol{j}+\boldsymbol{\theta}$, where $\theta$ has the azimuth $\phi$, so long as the particle is in Y. The velocity points for the particles with initial pitch angles ranging up to some small value $\theta_{0}$ lie within a small circular area of radius $\theta_{0}$ on the unit velocity sphere, centred on the point $-\boldsymbol{j}$. Each velocity point rotates round this centre with uniform angular speed $|e| \mathrm{H} / \mathrm{m}$. The circular area has the approximate magnitude $\pi \theta_{0}^{2}$.

In $z_{0}$ range $F\left(z_{0}>0.5\right)$ the path for $\theta_{0}=0$ returns to $Y$ from $Z$, and so do the adjacent paths of particles with small initial pitch angle. Finally their velocity points lie in a small area $\pi \theta_{0}^{2}$ centred on $j$. In $z_{0}$ range $\mathrm{N}\left(z_{0}<0.474\right)$ the path for $\theta_{0}=0$ extends from $Y$ through $Z$ and $-Y$ into $-Z$, and so do almost all the paths for particles with small initial pitch angle. Finally the velocity points describe circles round the point $-\boldsymbol{k}$ on the unit velocity sphere. The radius of this circle depends on $z_{0}$. For a fixed value of $z_{0}$ the velocity points for the paths where they cross any chosen plane $z=$ constant (negative) in $-Z$ will lie within a small area surrounding the velocity point for the particle with zero initial pitch angle where its path crosses this plane. The boundary of this area will be nearly circular. In our former paper [8] we found that the ratio of its area to $\pi \theta_{0}^{2}$ is of order unity.

The study of the paths in this very simple field distribution is helpful as a step towards consideration of the paths in a continuous field distribution near a region of low intensity, where $R / L$ becomes large. The paths in the present simple model field much resemble those in the continuous model field, and the similarity provides useful guidance for the classification of the paths in the continuous field.

\section{Scattering to low pitch angles in the field around a neutral line}

We next consider the hyperbolic field defined, relative to axes like those of Figure 1 , by

$$
\boldsymbol{H}=H \boldsymbol{h}=\left(\frac{H_{1}}{a}\right)(-y \boldsymbol{j}+z \boldsymbol{k}) .
$$

This field is illustrated by Figure 3 . The $x$-axis is a neutral line $(H=0)$. Elsewhere

$$
H=\frac{H_{1} r}{a}, \quad r^{2}=x^{2}+y^{2},
$$

so that $H_{1}$ is the field strength anywhere on the cylinder $r=a$. The field lines are the rectangular hyperbolas

$$
y z=C(\text { constant }) .
$$

They include as special cases the rectilinear field lines $C=\mathbf{0}$. 
As curl $\boldsymbol{H}=0$, the field (3.1) is free from electric current flow. Its scale length $L$, as defined by CHAPMAN [9], is given by

$$
L=\frac{r}{\sqrt{2}}
$$

The equation of motion of a charged particle subject only to the magnetic field,

$$
m \dot{\boldsymbol{v}}=e \boldsymbol{v} \times \boldsymbol{H},
$$

has the Cartesian components

$$
(\ddot{x}, \ddot{y}, \ddot{z})=\left(\frac{e H_{1}}{m a}\right)(y \dot{z}-z \dot{y},-\dot{x} z,-\dot{x} y) .
$$

We define new units of length and time, $L_{0}, T_{0}$, in terms of the measures initially used, by

$$
L_{0}=\left(R_{1} a\right)^{1 / 2}, \quad T_{0}=\frac{L}{v}, \quad R_{1}=\frac{m v}{|e| H_{1}},
$$

so that $R_{1}$ is the Larmor radius for such a particle moving transverse to a uniform field of strength $H_{1}$. In terms of these units

$$
v=1
$$

and the equations (3.6) for a positively charged particle become:

$$
\ddot{x}=\dot{y} z-\dot{z} y, \quad \ddot{y}=-\dot{x} z, \quad \ddot{z}=-\dot{x} y \text {. }
$$

If the charge is negative, a minus sign must be added on one side of each equation (3.9); this can be removed by reversing the direction of the $x$-axis (so that the axes become left handed). The paths for a negative particle are the mirror images, in any plane $x=$ constant, of those of a positively charged particle of the same charge/mass ratio. Hence it suffices to consider only the path of positively charged particles.

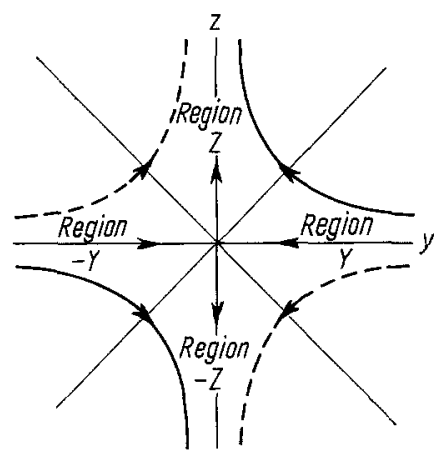

Figure 3

The hyperbolic magnetic field near a straight neutral line 
The first integrals of (3.9) are

$$
v=1, \dot{x}=y z-A,
$$

where $A$ denotes a constant of integration. These equations can be solved analytically [10] for particles initially moving in either of the planes $y= \pm z$; the paths lie wholly in the plane, with pitch angle everywhere $90^{\circ}$. The paths may be either wavy or looped; the mean motion is parallel to $\mathrm{O} x$. The motions are unstable; a small impulse given to the particle, perpendicular to the plane, leads to acceleration away from the plane.

In the general case $(3.10)$ gives

$$
\dot{y}^{2}+\dot{z}^{2}=1-(y z-A)^{2}=(1-y z+A)(1+y z-A) .
$$

As the left-hand side is positive or zero, the path for a given value of $A$ must lie between the hyperbolic cylinders

$$
y z=A \pm 1
$$

The allowed regions are of two types, shown in Figure 4 for the cases $A>1$ and $A<1[10]$.

Here, as in $\S 2$, we consider the paths of a particle that is initially in the upper half of the region $Y$ (defined as in Figure 1), spiralling leftward about a field line (3.3) for which $C$ is small. The Larmor radius $R$ is now not constant, as it was in $\S 2$; here it is given by

$$
R=\left(\frac{m v}{e H}\right) \sin \theta=\left(\frac{m a v}{e H_{1} v}\right) \sin \theta .
$$

Hence, using the units (3.7),

$$
R=\left(\frac{L_{0}^{2}}{r}\right) \sin \theta
$$

and

$$
\frac{R}{L}=2^{1 / 2} \frac{\sin \theta}{\gamma^{2}} .
$$
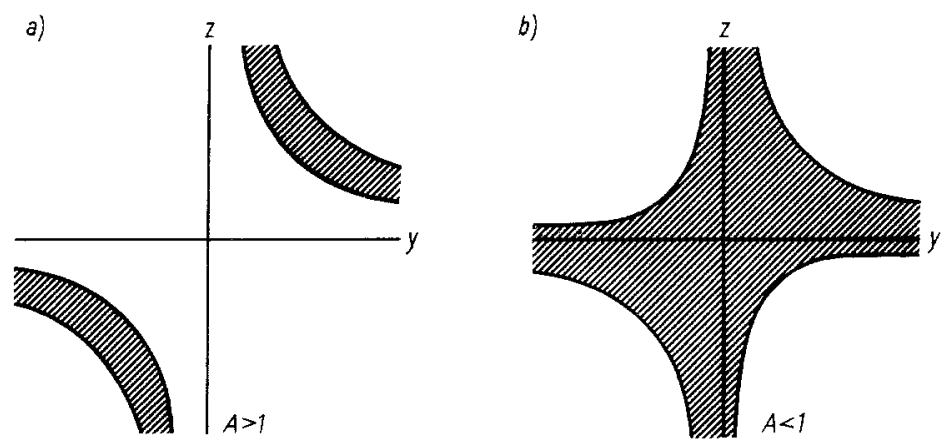

Figure 4

Allowed regions for particles moving in either of the planes $y= \pm z$ in the magnetic field of Figure 3 (Åsтröm [10]) 
Thus when $r$, expressed in the length unit (3.7), is large, $R / L$ is small and the motion of the particle is adiabatic; it can be adequately described in the way originated by ALFVÉN [1], as Larmorian motion about a guiding or Alfvén centre that moves nearly along a field line.

To be definite, we consider the motion of particles only within the region bounded by the four planes

$$
y= \pm a_{0}, \quad z= \pm a_{0}, \quad a_{0}=3 \sqrt{2}=4.243, \quad a_{0}^{2}=18
$$

(This choice of $a_{0}$ is somewhat arbitrary, and slightly affects the limits of the various. $z_{0}$ range intervals later discussed.) This will be called the NA (non-adiabatic) region; outside it,

$$
\frac{R}{L} \leq \frac{2^{3 / 2}}{18}=\frac{1}{12.7}
$$

there the motion can be considered adequately adiabatic, and conforming closely to the Alfvén description. Of course the non-adiabaticity within the NA region increases. towards the $x$-axis, and tends to effective adiabaticity near the boundary. There is. no loss of generality in taking the point $P_{0}$ of entry of a particle to be from the upper parts of region $\mathrm{Y}$ in the plane $x=0$; thus at entry $y=a_{0}$. We consider only small values of $z_{0}$, the $z$-coordinate of $P_{0}$. Thus $P_{0}$ is the point $\left(0, a_{0}, z_{0}\right)$. For small values of $z_{0}$ the path traverses the region where the field is weak and $R / L$ is large, so that the motion is non-adiabatic. Such paths, of course, lie in the 'allowed' region shown in Figure $4 \mathrm{~b}$.

Outside the NA region, where the field strength tends to infinity with increasing. distance from the $x$-axis, any particle emerging from within the NA region must move into stronger field and must have a mirror point there; hence it will return; similarly any particle entering the NA region must come from a region of stronger field. The paths that enter the NA region with a value of $z_{0}$ nearly equal to $a_{0}$ will be of spiral form between mirror points $\left(0, y_{1}, z_{1}\right)$ and $\left(0, z_{1}, y_{1}\right)$ on one of the outermost field lines that traverse the NA region.

\section{$3 a$ The nature of the non-adiabatic paths with zero pitch angle at $y=a_{0}$}

The fields illustrated in Figures 1 and 3 are in some respects similar. Both have four quadrantal regions, separated by the planes $y=0, z=0$; in each quadrant all the lines of force are similar in character and separate from those in the other quadrants. In each quadrant they turn through $90^{\circ}$, from the direction of the $y$-axis to that of the z-axis. One great difference, however, is that the field of Figure 1 is of finite intensity everywhere, and the paths in it have no mirror points; they all come from and go to infinity. In the field of Figure 3 all the paths except those with directions $\pm j$ in the plane $z=0$, or with the directions $\pm \boldsymbol{k}$ in the plane $y=0$, lie at finite distance from $\mathrm{O} x$, because of the unlimited outward increase of field intensity. The paths in the field of Figure 1 can be treated analytically; those in the field of Figure 3 have to be computed numerically.

Let $P_{0}, P_{1}$, at $\left(0, a_{0}, z_{0}\right)$ and $\left(x_{1}, y_{1}, z_{1}\right)$ respectively, be the points of entry and exit of the paths that traverse the NA region, and that have zero pitch angle at $P_{0}$.. 
At $P_{0}$ the sign of $\dot{r}$ is negative, at $P_{1}$ it is positive; thus there must be at least one reversal of sign of $r$ within the NA region; there may be more than one. The exit may be across the boundary in the $-Y$ region, in which case $y_{1}=-a_{0}$; if the exit is into the $Z$ region, $z_{1}=a$, and if into the $-Z$ region, $z_{1}=-a_{0}$. The simplest path is that for which $z_{0}=0$; it lies wholly along the $y$-axis, and the exit is into the region $-Y$; the time of passage through the NA region is $2 a_{0}$, in terms of the time unit defined by $(3.7)$.

We find that the paths have somewhat the same character is those of $\S 2$. The chief difference is that in $\S 2$ all paths of particles projected inwards with zero initial pitch angle in the upper part of region $Y$ eventually continue to infinity in one of the regions $Y,-Y,-Z$. For the continuous field the motion of particles is from one mirror point to another, and a particle may not emerge from the NA region until it has suffered many reflections. For small pitch angles these multiply reflected paths are confined to two ranges of $z_{0}, \mathrm{M}_{1}$ and $\mathrm{M}_{2}$, separating modified $z_{0}$ ranges $\mathrm{N}$ (near), I (intermediate) and $\mathrm{F}$ (far) in which the paths are similar to those in the corresponding ranges (2.4), (2.5) and (2.6). The various ranges of $z_{0}$ and the behaviour of particle paths are as follows.

$$
z_{0} \text { range } \mathrm{N} ; \text { for } 0 \leq z_{0} \leq Z_{0}, Z_{0} \doteqdot 0.0475
$$

The exit region is $-\mathrm{Y}$, and as $z_{0}$ increases from 0 to $Z_{0}$ the exit pitch angle steadily increases to $90^{\circ}$; the time of passage also increases steadily. The paths diverge more and more from the straight path for $z_{0}=0$, as $z_{0}$ increases up to the value $Z_{0}$. The precise numerical value of $Z_{0}$ must, of course, depend on the arbitrary choice of the number $a_{0}$. In this small initial range of $z_{0}$ the paths remain rather simple.

$$
z_{0} \text { range } \mathrm{M}_{1} ; \quad Z_{0}<z<Z_{1}, \quad Z_{1} \doteqdot 0.0975 \text {. }
$$

The radial velocity $\dot{r}$ is negative as the path initially crosses the boundary of the NA region, but $\dot{r}$ changes sign many times before the path again crosses the boundary. We refer to this $z_{0}$ range as one of multiple reflection, and to this type of path as multiply reflected.

$$
z_{0} \text { range } I ; \quad Z_{1} \leq z_{0} \leq Z_{2}, \quad Z_{2} \doteqdot 0.1085
$$

These paths through the NA region are simple and $\dot{r}$ changes sign once. The direction of mean motion is turned through a right angle and the exit is always into the $-Z$ region. For $z_{0}=Z_{1}$ the exit pitch angle is $90^{\circ}$. As $z_{0}$ increases, the exit pitch angle decreases monotonically to the value zero at $z_{0} \doteqdot 0.104$. As $z_{0}$ increases further, the exit pitch angle increases to $90^{\circ}$ at $z_{0}=Z_{2}$.

$$
z_{0} \text { range } \mathrm{M}_{2} ; \quad Z_{2}<z_{0}<Z_{3}, \quad Z_{3} \doteqdot 0.245
$$

The paths are multiply reflected.

$$
z_{0} \text { range } \mathrm{F} ; \quad Z_{3} \leq z_{0}<\infty .
$$

These paths through the NA region are simple and $\dot{r}$ changes sign once. The direction of mean motion is turned through a right angle and the exit is always into the $Z$ 
region. For $z_{0}=Z_{3}$ the exit pitch angle is $90^{\circ}$, and as $z_{0}$ increases the exit pitch angle decreases monotonically, becoming nearly zero for even moderately large values of $z_{0}$. These paths follow the curvature of the lines of force, and for large $z_{0}$ the motion is adequately described by the Alfvén approximation.

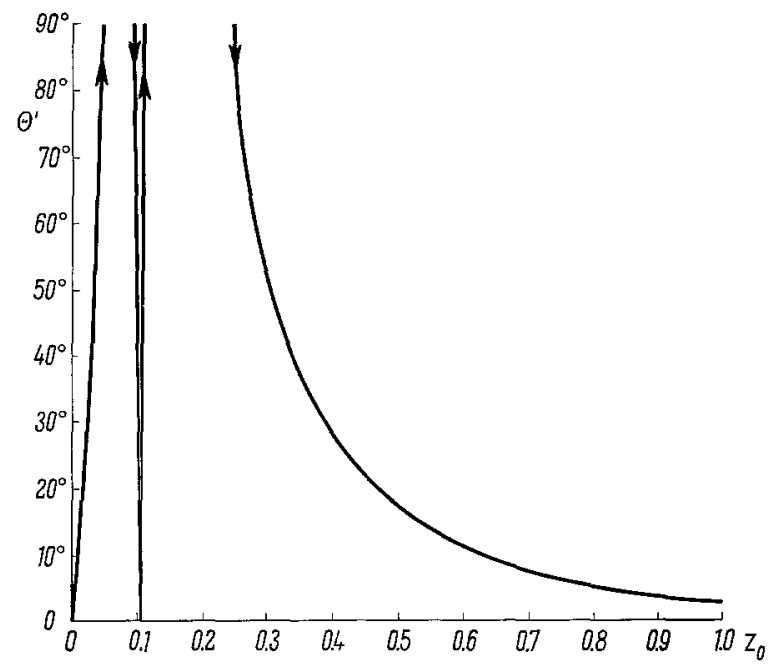

Figure 5

The variation of exit pitch angle $\theta^{\prime}$ with respect to $z_{0}$, for simple paths

Figure 5 illustrates the variation of exit pitch angle $\theta^{\prime}$ with $z_{0}$ for simple paths. No entry appears for the $z_{0}$ ranges of multiple reflection, on whose boundaries $\theta^{\prime}$ approaches $90^{\circ}$. Figure 6 shows the magnetic moment, $\left(\sin ^{2} \theta\right) / r$, in dimensionless variables, after ten units of time, as a function of $z_{0}$. In this time the non-multiply reflected paths emerge from the NA region. In the $z_{0}$ ranges of multiple reflection the curve is shown as a broken line. Two points are worth noting about Figure 6 , in which the $z_{0}$ scale is magnified tenfold compared with Figure 5. First, in the $z_{0}$ range I $\left(Z_{1} \leq z_{0} \leq Z_{2}\right)$ there is near-symmetry about $z_{0}=0.104$. Second, the magnetic moment in the $z_{0}$ region $\mathrm{M}_{1}$ of multiple reflection varies rapidly, and the broken curve merely connects the calculated points, and elsewhere may not show the true value of $\mu$.

Table 1 records the exit value of the number of reflections, the region of exit, the exit pitch angle $\theta^{\prime}$ and azimuth $\phi^{\prime}$, and the exit time, for various values of $z_{0}$.

This table illustrates one of the little understood results obtained by workers who calculate the paths of charged particles in magnetic mirror machines. Even though multiply reflected paths are very complicated there is sometimes a tendency for a certain apparent regularity in the type of path to appear. This is shown particularly in the entries for $z_{0}=0.11,0.15$ and 0.2 . The corresponding paths are looped in various degrees, but the exit region is always the same. The reason for this is unknown; the change from Åström's [10] paths of Figure $4 \mathrm{a}$ to the different type of Figure $4 \mathrm{~b}$ is expected at $z_{0} \doteqdot 0.24$. Table 1 also shows clearly the different types of simple path obtained. 
Table 1

Results for paths with zero initial pitch angle

\begin{tabular}{|c|c|c|c|c|c|c|}
\hline$z_{0}$ & $\begin{array}{l}\text { In } z_{0} \\
\text { range }\end{array}$ & $\begin{array}{l}\text { Number of } \\
\text { reflections }\end{array}$ & $\begin{array}{l}\text { Region } \\
\text { of exit }\end{array}$ & $\begin{array}{l}\theta^{\prime} \\
\text { (degrees) }\end{array}$ & $\begin{array}{l}\phi^{\prime} \\
\text { (degrees) }\end{array}$ & Time \\
\hline .015 & $\mathrm{~N}$ & 0 & $-Y$ & 18.89 & -59.99 & 8.60 \\
\hline .025 & $\mathrm{~N}$ & 0 & $-\mathrm{Y}$ & 32.07 & 260.35 & 8.84 \\
\hline .035 & $\mathrm{~N}$ & 0 & $-Y$ & 48.61 & 170.11 & 9.36 \\
\hline .05 & $\mathrm{M}_{1}$ & 6 & $-Z$ & 23.39 & 42.53 & 61.69 \\
\hline .075 & $\mathrm{M}_{1}$ & 7 & $-Y$ & 23.07 & -31.52 & 47.76 \\
\hline .095 & $\mathrm{M}_{1}$ & 8 & $-Z$ & 58.28 & -69.15 & 79.28 \\
\hline .0975 & $\mathrm{M}_{1}$ & 1 & $-Z$ & 79.62 & -34.57 & 13.82 \\
\hline .1 & I & 0 & $-Z$ & 40.04 & 54.02 & 11.60 \\
\hline .104 & I & 0 & $-Z$ & 0.61 & 63.46 & 11.16 \\
\hline .1075 & I & 0 & $-Z$ & 41.93 & -132.89 & 11.95 \\
\hline .11 & $\mathrm{M}_{2}$ & 1 & Z & 36.44 & 20.92 & 22.81 \\
\hline .15 & $\mathrm{M}_{2}$ & 5 & $Z$ & 36.97 & 58.69 & 39.05 \\
\hline .2 & $\mathrm{M}_{2}$ & 3 & $Z$ & 50.95 & 219.53 & 26.01 \\
\hline .3 & $\mathrm{~F}$ & 0 & $Z$ & 50.11 & -27.42 & 7.82 \\
\hline .4 & $F$ & 0 & Z & 28.77 & 207.36 & 6.74 \\
\hline .5 & $\mathrm{~F}$ & 0 & $Z$ & 17.86 & 175.72 & 6.26 \\
\hline 1.0 & $\mathrm{~F}$ & 0 & Z & 4.04 & 48.71 & 4.97 \\
\hline
\end{tabular}

A numerical approximation to the exit pitch angle for $Z_{1} \leq z_{0} \leq Z_{2}$ is

accurate to $\pm 1^{\circ}$.

$$
\theta^{\prime}=2.9 \times 10^{6}\left(z_{0}-0.10371\right)^{2}
$$

$3 b$ The mature of the non-adiabatic paths with initial pitch angles of one degree at $y=a_{0}$

For these paths the pitch angle $\theta^{\prime}$ and azimuth $\phi^{\prime}$ at exit may depend greatly on the initial azimuth $\phi$. However, the region of exit for simple paths is the same as for zero initial pitch angle, except for small intervals close to $z_{0}=Z_{0}, Z_{1}, Z_{2}$ and $Z_{3}$.

Consider first the multiply reflected paths. These were investigated for 8 different lines of force or values of $z_{0}$ (Table 2), and the variation of the exit time, the number of reflections and the region of exit were noted for various values of the initial azimuth $\phi$. Paths that did not depart from the NA region after 100 units of time were classified as ue (unknown emergence). The accuracy of the numerical integration was periodically tested by the computer, and inaccurate paths were ignored; they are marked inacc in Table 2. The results obtained are summarized in Table 2. Of the 164 paths calculated, six are marked ue and two were inaccurate (these two were not recomputed). Table 3 shows the number of paths emerging into each region for different lines of force. It is clear that a much finer lattice of values of $z_{0}$ would be needed to exhibit the average behaviour of these multiply reflected paths. 
Table 2

Times of exit of multiply reflected paths with initial pitch angles of $1^{\circ}$. Above the value of the time of exit the exit region and number of reflections are given

\begin{tabular}{|c|c|c|c|c|c|c|c|c|}
\hline$\pi-\phi$ & $z_{0} \quad 0.05$ & 0.075 & 0.095 & 0.0975 & 0.1075 & 0.11 & 0.15 & 0.2 \\
\hline \multirow[t]{2}{*}{$0^{\circ}$} & $\begin{array}{ll}\mathrm{Y} & 1\end{array}$ & $Y \quad 3$ & Y 5 & $-\mathrm{Y} 6$ & $-Z \quad 0$ & $-Y 5$ & $\begin{array}{ll}-\mathrm{Y} & 1\end{array}$ & $\mathrm{Y}$ \\
\hline & 24.17 & 25.88 & 42.87 & 49.97 & 11.61 & 46.58 & 16.15 & 17.74 \\
\hline \multirow[t]{2}{*}{$20^{\circ}$} & $-\mathrm{Y} 9$ & $-Y 13$ & $-\mathrm{Y} 3$ & $-Z \quad 1$ & $-Z \quad 0$ & $-\mathrm{Y} 3$ & $-Z \quad 6$ & Z 3 \\
\hline & 65.80 & 66.25 & 22.64 & 14.07 & 11.88 & 24.28 & 56.85 & 29.01 \\
\hline \multirow[t]{2}{*}{$40^{\circ}$} & $Z \quad 1$ & Y 1 & Z 2 & $-Z \quad 1$ & $-Z \quad 0$ & $Y \quad 6$ & $-Z \quad 3$ & $-Z \quad 9$ \\
\hline & 26.80 & 16.70 & 23.85 & 12.77 & 12.33 & 49.07 & 22.76 & 75.27 \\
\hline \multirow[t]{2}{*}{$60^{\circ}$} & $-\mathrm{Y} 8$ & $\mathrm{Y} \quad 1$ & Z 10 & $-\mathrm{Z} \quad 0$ & $-Z \quad 1$ & $\mathrm{Y} 4$ & $-Z 3$ & \\
\hline & 66.61 & 15.17 & 96.02 & 12.24 & 13.10 & 33.12 & 20.63 & 48.47 \\
\hline \multirow[t]{2}{*}{$80^{\circ}$} & $-Y 10$ & Y 1 & Z 3 & $-Z \quad 0$ & & $Z \quad 1$ & $Y \quad 3$ & Z 2 \\
\hline & 73.07 & 15.19 & 41.78 & 12.00 & inacc & 18.51 & 21.05 & 22.35 \\
\hline \multirow[t]{2}{*}{$100^{\circ}$} & Y 1 & Y 1 & $-Y 4$ & $\begin{array}{ll}-Z & 0\end{array}$ & $Z 2$ & Z 4 & Z 3 & $-\mathrm{Y} 4$ \\
\hline & 22.46 & 15.41 & 41.34 & 11.89 & 27.67 & 29.55 & 24.43 & 38.90 \\
\hline \multirow[t]{2}{*}{$120^{\circ}$} & Y 1 & Y 1 & $Z 6$ & $-Z \quad \begin{array}{ll}Z & 0\end{array}$ & $-Z 2$ & Z 3 & $-Z 9$ & 13 \\
\hline & 22.51 & 15.40 & 58.74 & 11.88 & 32.78 & 28.11 & 83.42 & ue \\
\hline \multirow[t]{2}{*}{$140^{\circ}$} & $-\mathrm{Y} 5$ & Y 1 & 10 & $-Z \quad 0$ & Z 8 & Y 6 & Y 8 & $\mathrm{Y} \quad 2$ \\
\hline & 38.00 & 15.18 & ue & 11.97 & 62.44 & 46.06 & 46.50 & 23.95 \\
\hline \multirow[t]{2}{*}{$160^{\circ}$} & $Z \quad 2$ & Y 1 & $Z 2$ & $\begin{array}{ll}-\mathrm{Z} & 0\end{array}$ & $-Z \quad 1$ & Y 3 & $-Y 5$ & Y 8 \\
\hline & 29.13 & 15.20 & 25.32 & 1217 & 13.59 & 27.70 & 43.14 & 56.44 \\
\hline \multirow[t]{2}{*}{$180^{\circ}$} & 12 & $\mathrm{Y} \quad 2$ & $-\mathrm{Y} \quad 5$ & $-Z 0$ & $-Z \quad 1$ & Z 4 & $\mathrm{Y} \quad 6$ & $-Z \quad 5$ \\
\hline & ue & 17.15 & 39.05 & 12.60 & 12.61 & 34.24 & 43.04 & 38.55 \\
\hline \multirow[t]{2}{*}{$200^{\circ}$} & $-Z \quad 7$ & Z 11 & $-Z \quad 6$ & $-Z \quad 2$ & $-Z \quad 0$ & $-Z \quad 2$ & Z 4 & Z 2 \\
\hline & 52.75 & 84.19 & 54.94 & 13.63 & 12.04 & 28.22 & 24.88 & 22.40 \\
\hline \multirow[t]{2}{*}{$220^{\circ}$} & $-Z 3$ & Z 4 & $-Z 4$ & Y 13 & $-Z \quad 0$ & $-\mathrm{Y} 4$ & $-Z \quad 2$ & $-Z 11$ \\
\hline & 33.13 & 40.48 & 29.50 & 99.97 & 11.71 & 39.32 & 24.83 & 77.14 \\
\hline \multirow[t]{2}{*}{$240^{\circ}$} & 14 & Z 4 & $-Y 4$ & $-\mathrm{Y} \quad 4$ & $-\begin{array}{ll}Z & 0\end{array}$ & $\mathrm{Y} \quad 3$ & $-\mathrm{Y} \quad 2$ & \\
\hline & ue & 35.90 & 33.39 & 51.92 & 11.51 & 40.49 & 17.04 & inacc \\
\hline \multirow[t]{2}{*}{$260^{\circ}$} & $Y \quad 1$ & 14 & $-\mathrm{Y} 3$ & $-Z \quad 9$ & $-Z \quad 0$ & $-Z \quad 1$ & $-\mathrm{Y} \quad 1$ & Z 3 \\
\hline & 26.12 & ne & 19.47 & 73.90 & 11.40 & 13.16 & 15.91 & 29.23 \\
\hline \multirow[t]{2}{*}{$280^{\circ}$} & 9 & $-Z \quad 2$ & $-Z \quad 4$ & $Z \quad 11$ & $-Z \quad 0$ & $-Z \quad 1$ & $\begin{array}{ll}-Y & 1\end{array}$ & Y 3 \\
\hline & ue & 16.99 & 23.73 & 88.28 & 11.35 & 12.72 & 16.11 & 17.71 \\
\hline \multirow[t]{2}{*}{$300^{\circ}$} & Z 4 & $-Z \quad 2$ & $-Z 3$ & Z 4 & $-Z \quad \begin{array}{ll}Z & 0\end{array}$ & $-Z \quad 1$ & $\begin{array}{ll}-\mathrm{Y} & 2\end{array}$ & Y 1 \\
\hline & 56.54 & 17.04 & 22.64 & 40.56 & 11.34 & 12.65 & 16.55 & 16.83 \\
\hline \multirow[t]{2}{*}{$320^{\circ}$} & $-Z 3$ & $-7 \quad 3$ & $-\mathrm{Y} 3$ & $-Z 2$ & $-Z \quad 0$ & $\begin{array}{ll}Z & 1\end{array}$ & $-\mathrm{Y} \quad 2$ & $\mathrm{Y} \quad 1$ \\
\hline & 42.78 & 48.10 & 20.49 & 26.03 & 11.37 & 12.91 & 16.35 & 16.66 \\
\hline \multirow[t]{2}{*}{$340^{\circ}$} & Z 4 & $-Z 7$ & $-Y 3$ & $-Z \quad 6$ & $-Z \quad 0$ & $-Z \quad 1$ & $\begin{array}{ll}-Y & 1\end{array}$ & Y 1 \\
\hline & 39.17 & 46.27 & 29.15 & 42.81 & 11.45 & 13.78 & 15.92 & 16.84 \\
\hline
\end{tabular}

Table 3

The number of paths with initial pitch angle $1^{\circ}$ leading into each region

\begin{tabular}{|c|c|c|c|c|c|c|c|c|}
\hline Exit region $z_{0}$ & 0.05 & 0.075 & 0.095 & 0.0975 & 0.1075 & 0.11 & 0.15 & 0.2 \\
\hline$Y$ & 4 & 9 & 1 & 1 & 0 & 5 & 3 & 7 \\
\hline$Z$ & 4 & 3 & 5 & 2 & 2 & 4 & 2 & 5 \\
\hline$-Y$ & 4 & 1 & 7 & 2 & 0 & 3 & 8 & 1 \\
\hline$-Z$ & 3 & 4 & 4 & 13 & 15 & 6 & 5 & 3 \\
\hline ue or inacc & 3 & 1 & 1 & 0 & 1 & 0 & 0 & 2 \\
\hline
\end{tabular}


Vol. 59, 1964/III) Charged Particles Traversing a Weak Magnetic Field

Table 4

Emergent pitch $\theta^{\prime}$ in degrees for paths entering with $1^{\circ}$ pitch angle.

The entries are recorded relative to the results for zero pitch

\begin{tabular}{|c|c|c|c|c|c|c|c|c|c|c|}
\hline $\begin{array}{l}z_{0} \\
z_{0} \text { range }\end{array}$ & $\begin{array}{l}0.015 \\
\mathrm{~N}\end{array}$ & $\begin{array}{l}0.025 \\
\mathrm{~N}\end{array}$ & $\begin{array}{l}0.035 \\
\mathrm{~N}\end{array}$ & $\begin{array}{l}0.1 \\
\mathrm{I}\end{array}$ & $\begin{array}{l}0.104 \\
\mathrm{I}\end{array}$ & $\begin{array}{l}0.1075 \\
\mathrm{I}\end{array}$ & $\begin{array}{l}0.3 \\
F\end{array}$ & $\begin{array}{l}0.4 \\
F\end{array}$ & $\begin{array}{l}0.5 \\
F\end{array}$ & $\begin{array}{l}1.0 \\
F\end{array}$ \\
\hline \multicolumn{11}{|l|}{$\begin{array}{l}\text { Results } \\
\text { for zero }\end{array}$} \\
\hline pitch & 18.89 & 32.07 & 48.61 & 40.04 & 0.61 & 41.93 & 50.11 & 28.77 & 17.86 & 4.04 \\
\hline$\phi=0^{\circ}$ & 0.67 & 0.72 & 1.09 & $\begin{array}{l}-6.69 \\
\end{array}$ & 6.20 & - & -1.39 & -0.96 & -0.89 & -0.55 \\
\hline $20^{\circ}$ & 1.43 & 1.52 & 2.31 & -11.63 & 11.85 & - & -1.53 & -1.05 & -0.99 & -0.81 \\
\hline $40^{\circ}$ & 2.01 & 2.14 & 3.24 & -15.22 & 16.04 & - & -1.49 & -1.01 & -0.97 & -0.96 \\
\hline $60^{\circ}$ & 2.35 & 2.49 & 3.78 & -16.97 & 18.08 & - & -1.28 & -0.84 & -0.82 & -0.96 \\
\hline $80^{\circ}$ & 2.40 & 2.55 & 3.85 & -16.65 & 17.60 & - & -0.92 & -0.57 & -0.57 & -0.81 \\
\hline $100^{\circ}$ & 2.16 & 2.29 & 3.47 & -14.3 & 14.71 & - & -0.44 & -0.23 & -0.26 & -0.54 \\
\hline $120^{\circ}$ & 1.66 & 1.75 & 2.67 & -10.29 & 9.93 & - & 0.11 & 0.14 & 0.09 & -0.21 \\
\hline $140^{\circ}$ & 0.96 & 1.01 & 1.54 & $-\quad 5.11$ & 4.00 & 9.89 & 0.67 & 0.48 & 0.38 & 0.14 \\
\hline $160^{\circ}$ & 0.16 & 0.16 & 0.24 & 0.88 & 1.10 & -2.00 & 1.17 & 0.77 & 0.71 & 0.45 \\
\hline $180^{\circ}$ & -0.66 & -0.70 & -1.06 & 7.91 & 7.09 & -10.24 & 1.53 & 0.97 & 0.91 & 0.71 \\
\hline $200^{\circ}$ & -1.39 & -1.47 & -2.22 & 14.88 & 12.18 & -16.74 & 1.71 & 1.05 & 1.00 & 0.89 \\
\hline $220^{\circ}$ & -1.95 & -2.07 & -3.09 & 19.33 & 15.81 & -21.17 & 1.68 & 1.01 & 0.98 & 0.98 \\
\hline $240^{\circ}$ & -2.28 & -2.41 & -3.59 & 21.48 & 17.56 & -23.21 & 1.44 & 0.85 & 0.85 & 0.98 \\
\hline $260^{\circ}$ & -2.35 & -2.47 & -3.67 & 21.14 & 17.23 & -22.74 & 1.02 & 0.59 & 0.61 & 0.88 \\
\hline $280^{\circ}$ & -2.13 & -2.23 & -3.33 & 18.48 & 14.84 & -19.76 & 0.49 & 0.26 & 0.31 & 0.69 \\
\hline $300^{\circ}$ & -1.65 & -1.72 & -2.60 & 13.26 & 10.66 & -14.44 & -0.09 & -0.11 & -0.04 & 0.43 \\
\hline $320^{\circ}$ & -0.97 & -1.00 & -1.53 & 5.95 & 5.15 & -7.18 & -0.64 & -0.46 & -0.38 & 0.12 \\
\hline $340^{\circ}$ & -0.16 & -0.15 & -0.25 & $-\quad 0.86$ & -0.08 & 2.21 & -1.08 & -0.76 & -0.68 & -0.22 \\
\hline
\end{tabular}

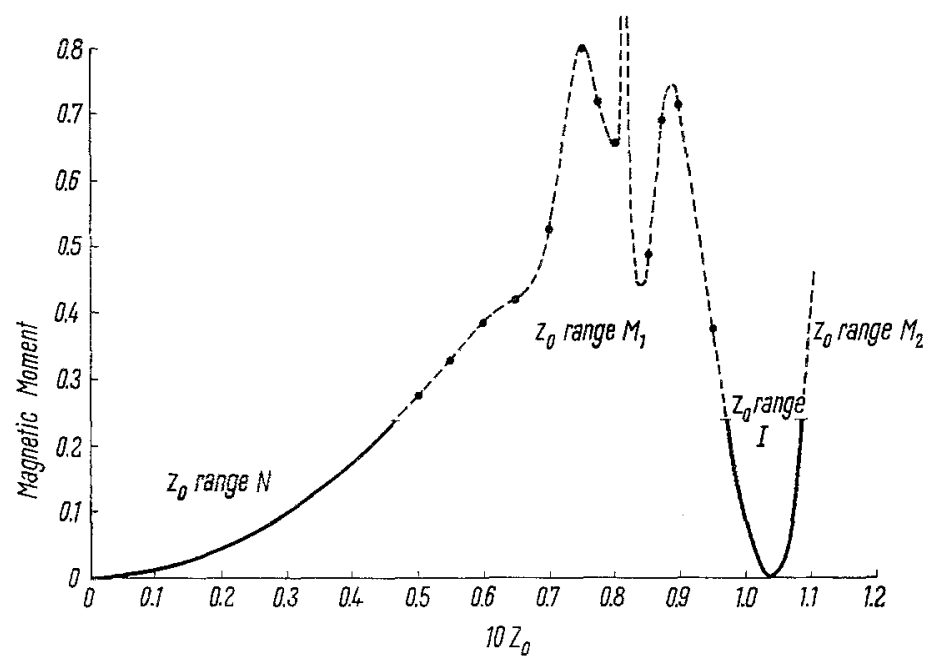

Figure 6

The magnetic moment after ten units of time, as a function of $z_{0}$ 
Table 5

Emergent azimuth $\phi^{\prime}$ in degrees for paths entering with $1^{\circ}$ pitch angle. The entries are recorded relative to the results for zero pitch

$\begin{array}{lllllllllll}z_{0} & 0.015 & 0.025 & 0.035 & 0.1 & 0.104 & 0.1075 & 0.3 & 0.4 & 0.5 & 1.0 \\ z_{0} \text { range } & \mathrm{N} & \mathrm{N} & \mathrm{N} & \mathrm{I} & \mathrm{I} & \mathrm{I} & \mathrm{F} & \mathrm{F} & \mathrm{F} & \mathrm{F}\end{array}$

\section{Results}

for zero

\begin{tabular}{|c|c|c|c|c|c|c|c|c|c|c|c|}
\hline pitch & -59.99 & 260.35 & 170.11 & & 54.02 & 63.46 & -132.89 & -27.42 & 207.36 & 175.72 & 48.71 \\
\hline$\phi=0^{\circ}$ & 0.63 & --1.73 & $-\quad 6.17$ & & 33.56 & -88.33 & - & -11.15 & -2.27 & -0.44 & -12.32 \\
\hline $20^{\circ}$ & -0.43 & -4.87 & -13.98 & & 53.05 & -94.08 & - & -12.94 & -3.28 & -1.80 & -8.41 \\
\hline $40^{\circ}$ & -1.49 & -7.53 & -20.32 & & 63.63 & -100.32 & - & -13.22 & -3.89 & -2.95 & 2.67 \\
\hline $60^{\circ}$ & -2.33 & -9.24 & -24.18 & & 68.02 & 104.04 & - & -11.96 & -4.03 & -3.72 & 3.77 \\
\hline $80^{\circ}$ & -2.81 & -9.74 & -24.90 & & 67.33 & -103.19 & - & 9.26 & -3.66 & -4.00 & 9.34 \\
\hline $100^{\circ}$ & -2.89 & -8.96 & -22.38 & & 61.34 & -98.34 & - & 5.37 & -2.84 & -3.77 & 12.92 \\
\hline $120^{\circ}$ & -2.11 & -7.05 & -17.10 & & 48.72 & -92.23 & - & 0.71 & -1.66 & -3.07 & 13.46 \\
\hline $140^{\circ}$ & -2.09 & -4.40 & $-\quad 9.95$ & & 27.08 & -88.00 & -64.33 & 4.19 & -0.28 & -1.99 & 13.69 \\
\hline $160^{\circ}$ & -1.49 & -1.42 & $-\quad 2.01$ & - & 5.01 & 93.83 & 12.01 & 8.70 & 1.14 & -0.70 & 11.66 \\
\hline $180^{\circ}$ & -0.88 & 1.49 & 5.73 & - & 47.36 & 90.04 & 52.13 & 12.21 & 2.42 & 0.66 & 8.64 \\
\hline $200^{\circ}$ & -0.31 & 4.00 & 12.41 & - & 93.98 & 84.41 & 72.55 & 14.23 & 3.40 & 1.92 & 5.01 \\
\hline $220^{\circ}$ & 0.24 & 5.94 & 17.44 & - & 119.27 & 78.71 & 82.44 & 14.48 & 3.98 & 2.95 & 1.09 \\
\hline $240^{\circ}$ & 0.80 & 7.08 & 20.40 & - & 272.19 & 75.40 & 86.05 & 12.94 & 4,08 & 3.64 & $-\quad 2.87$ \\
\hline $260^{\circ}$ & 1.35 & 7.46 & 21.06 & - & 266.95 & 75.96 & 85.32 & 9.85 & 3.70 & 3.93 & $-\quad 6.62$ \\
\hline $280^{\circ}$ & 1.82 & 7.18 & 19.39 & 一 & 128.65 & 80.12 & 79.73 & 5.63 & 2.99 & 3.77 & -9.1 \\
\hline $300^{\circ}$ & 2.07 & 6.03 & 15.41 & - & 82.10 & 85.88 & 66.35 & 0.85 & 1.75 & 3.18 & -12.49 \\
\hline $320^{\circ}$ & 1.97 & 4.09 & 9.43 & - & 35.58 & 90.41 & 38.97 & - $\quad 3.88$ & 0.40 & 2.21 & -14.02 \\
\hline $340^{\circ}$ & 1.48 & 1.40 & 1.97 & & 4.79 & -75.75 & -13.80 & -8.03 & -0.99 & 0.96 & -14.10 \\
\hline
\end{tabular}

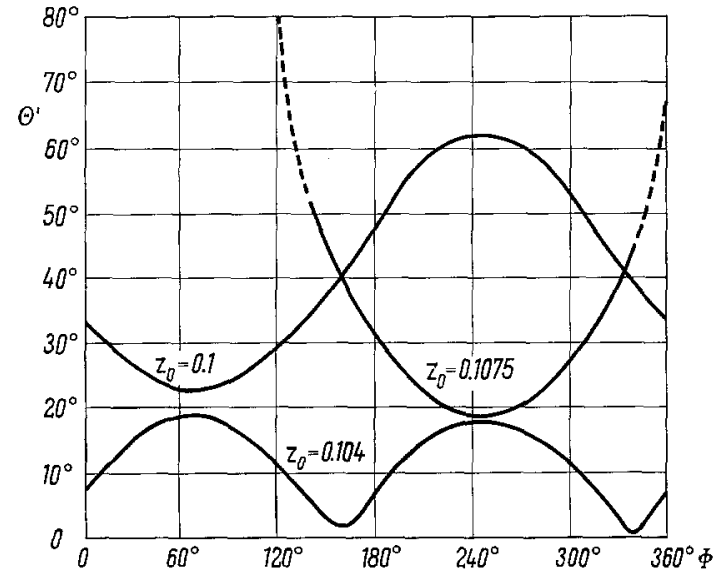

Figure 7

Variations of the exit pitch angle $\theta^{\prime}$ with respect to the initial azimuth $\phi$, for initial pitch angles of $1^{\circ}$ and for three values of $z_{0}$ in the I range $\left(Z_{1} \leq z_{0} \leq Z_{2}\right)$ 
Tables 4 and 5 show for the simpler paths the emergent pitch angles $\theta^{\prime}$ and azimuthal angles at the exit point for various lines of force. For each value of $z_{0}$ the results for zero initial pitch angle and azimuth are given at the head of the column. Three values of $z_{0}$ in the $z_{0}$ range $\mathrm{N}\left(0 \leq z_{0} \leq Z_{0}\right)$ were chosen, three in the $z_{0}$ range $\mathrm{I}\left(Z_{1} \leq z_{0} \leq Z_{2}\right)$ and four in the $z_{0}$ range $\mathrm{F}\left(Z_{3} \leq z_{0}<\infty\right)$. In the case $z_{0}=0.1075$ some of the paths are of the multiply reflected type, and for such paths no value of $\theta^{\prime}$ is recorded. Figures 7 and 8 show the variation of $\theta^{\prime}$ and $\phi^{\prime}$ for three values of $z_{0}$ in the $z_{0}$ range $\mathrm{I}\left(Z_{1} \leq z_{0} \leq Z_{2}\right)$. The variation of $\phi^{\prime}$ is very rapid for certain values of the initial azimuth $\phi$ for the paths for $z_{0}=0.104$. There is a corresponding near-cusp on the $\theta^{\prime}$ curve. On the velocity sphere the corresponding points trace out a curve that passes near the pole. As the curve passes the pole, $\phi^{\prime}$ alters by $180^{\circ}$. We note also, as indicated in Figure 6, that near $z_{0}=0.104$ there exists a symmetric orbit whose initial pitch angle is zero and whose exit pitch angle is also zero.

\section{3c The width of a particle beam at its point of exit from the $N A$ region}

We are interested in the change in density of a narrow beam of particles injected at $z=z_{0}$ with pitch angles of less than one degree, when the beam reaches the edge of the NA region. This may be evaluated by comparing the solid angles filled by the velocity vectors initially and at exit. Denote by $P$ the point on the velocity sphere corresponding to the initial state and by $P^{\prime}$ the point corresponding to the state at exit from the NA region. As $P$ describes a small circle corresponding to $\theta=1^{\circ}, P^{\prime}$ describes some closed curve $C$ on the velocity sphere. The point $O$ corresponding to zero initial pitch angle will lie somewhere within $C$. For example, Figure 9 shows the curve $C$ and the point $O$ for $z_{0}=0.015$. The curve is drawn in the $\left(\theta^{\prime}, \phi^{\prime}\right)$ plane, because its dimensions are small on the velocity sphere. The point $A$ corresponds to $\phi=0$. As $\phi$ increases from 0 to $360^{\circ}$ in steps of $20^{\circ}$, the points $\left(\theta^{\prime}, \phi^{\prime}\right)$ move round the curve in the direction of the arrow.

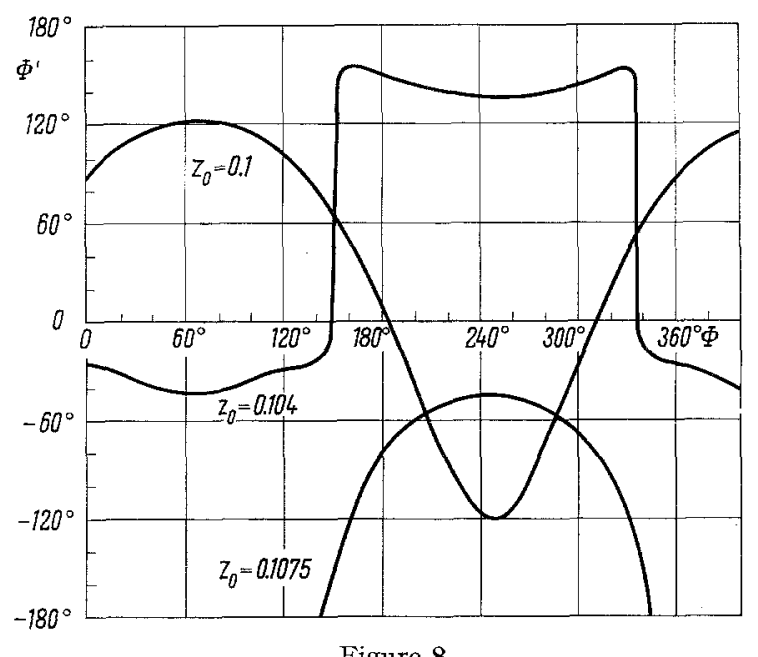

Figure 8

Variations of the exit azimuthal angle $\phi^{\prime}$ with respect to the initial azimuth $\phi$, for initial pitch angles of $1^{\circ}$, ans for three values of $z_{0}$ in the $I$ range $\left(Z_{1} \leq z_{0} \leq Z_{2}\right)$ 
The solid angle ratio $\mathfrak{R}$ is defined by

$$
\left(\frac{\pi^{3}}{180^{2}}\right) \Re=\left|\iint_{S} d\left(\cos \theta^{\prime}\right) d \phi^{\prime}\right|,
$$

where $S$ denotes the interior of $C$. This is proportional to the area in the $\left(\cos \theta^{\prime}, \phi^{\prime}\right)$ plane. If the values of $\theta^{\prime}$ deviate only a little from a fixed value $\theta_{0}^{\prime}$ (the value of $\theta^{\prime}$ for zero initial pitch) we may approximate, giving

$$
\left(\frac{\pi^{3}}{180^{2}}\right) \Re=\left|\sin \theta_{0}^{\prime} \iint_{S} d \theta^{\prime} d \phi^{\prime}\right| .
$$

The above areas were found in all cases possible. The case $z_{0}=0.035$ illustrates one difficulty encountered. The contour $C$, as given by Tables 4 and $\mathbf{5}$, is $\mathbf{5 0 0}$ times longer than its own width. The $\left(\cos \theta^{\prime}, \phi^{\prime}\right)$ diagram was therefore drawn, but magnified by using various coordinate transformations. It was necessary to treat the cases $z_{0}=0.035$, 0.3 in this way. It was not possible to deal in this way with paths in region $\mathrm{I}\left(Z_{1} \leq z_{0}\right.$ $\leq Z_{2}$ ) because the area was too long and thin. In the case of the $z_{0}$ ranges of multiple reflection $\mathrm{M}_{1}$ and $\mathrm{M}_{2}$ not enough detail was available. Table 6 shows the only values of $\Re$ calculated. These are for $z_{0}$ ranges $N\left(0 \leq z_{0} \leq Z_{0}\right)$ and $\mathrm{F}\left(Z_{3} \leq z_{0}<\infty\right)$. We may conjecture, by comparison with the discontinuous field model of $\S 2$, that $\Re=1$ near $z_{0}=0.104$, where there is a symmetric path. Figure 10 shows the interesting shape of the narrow curve $C$ for $z_{0}=0.104$. The curve is shown as if viewed from above the pole of the unit velocity sphere. Note the lateral magnification which is needed to exhibit the features of the curve.

Table 6

The solid angle ratio $\mathfrak{R}$ for various values of $z_{0}$

\begin{tabular}{llllllll}
\hline$z_{0}$ & 0.015 & 0.025 & 0.035 & 0.3 & 0.4 & 0.5 & 1.0 \\
$\mathfrak{R}$ & 1.04 & 1.15 & 1.44 & 2.02 & 1.12 & 1.01 & 1.00 \\
\hline
\end{tabular}

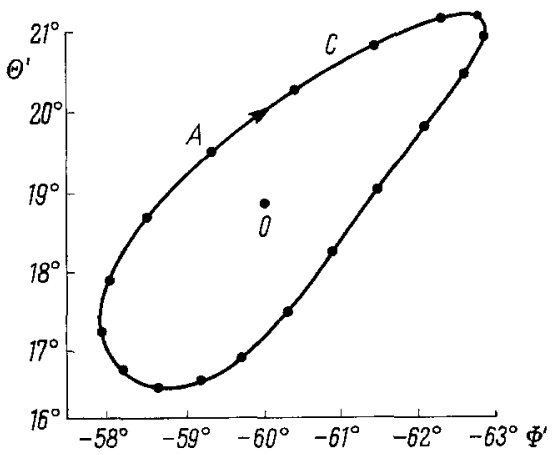

Figure 9

The closed curve $C$ on the velocity sphere for $z_{0}=0.015$. The point $O$ corresponds to zero initial pitch angle. The curve $C$ corresponds to an initial pitch angle of $1^{\circ}$, for different initial azimuthal angles 


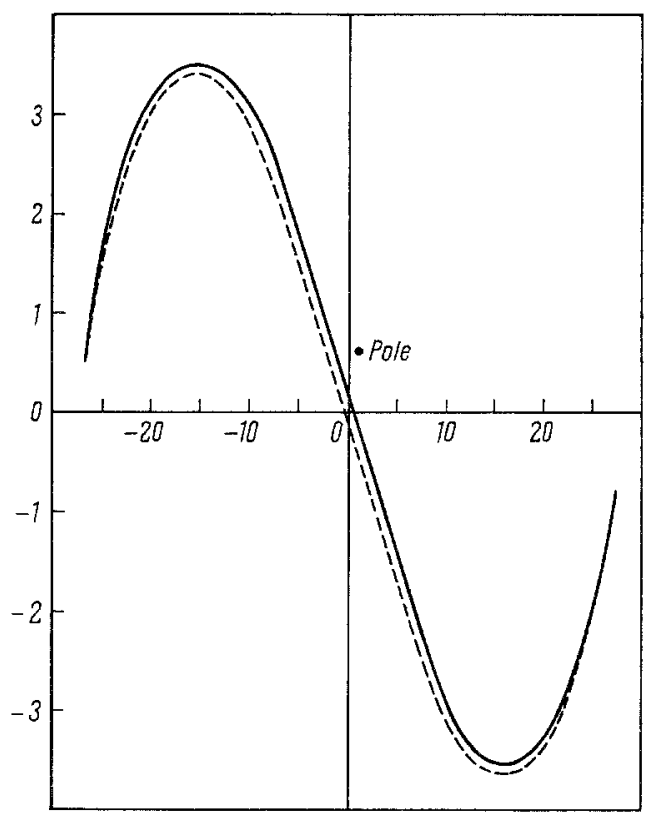

Figure 10

The curve $C$ for $z_{0}=0.104$

4. The case when the magnetic field is reduced nearly to zero

A less extensive investigation has been carried out for the case when the magnetic field does not vanish, but decreases nearly to zero. The magnetic field considered was

$$
\boldsymbol{H}=\left(0, D z, E+F y^{2}\right) \text {. }
$$

Figure 11 shows some of the field lines for the values $D=1, E=1, F=3$. We assume that $E \geq 0$. The configuration is then known as a minimum $B$ configuration [11]. As $E$ approaches zero the curvature of the lines of force at the origin becomes smaller, until for $E=0$ the field line that passes through the origin has a cusp there. For $E<0$ there would be two neutral lines of different types, as indicated by MCDONALD [12].

\section{4a. The paths of charged particles}

The $z$-range of the non-adiabatic (NA) region in this case is taken to be $|z| \leq 3$. Consider the motion of particles projected with zero pitch angle into the NA region from the plane $z=-3$. The velocity of projection is taken to be one unit. A qualitative estimate of the non-adiabaticity of a path is the time $\tau$ (in units of $e / m$ ) taken for $|z|$ to return to its initial value 3 . Adiabatic paths give values of $\tau$ close to 6 . Figure 12 shows $\tau$ as a function of $y_{0}$ for values $E=0.0,0.2,0.4$, with $D=1, F=3$. It is clear that much pitch angle scattering is occurring. Further values of $E$ were 
investigated up to $E=1$ without much change in the scattering effect. For interest, the path shown in Figure 13 was calculated in great detail for $E=1, y_{0}=1.3$. The path does not emerge from the NA region within 123 units of time. This is 20 times longer than the corresponding time for an adiabatic path. Figure 13 shows only the first part of the path. Eventually the particle emerges from the NA region with a velocity antiparallel to its initial velocity. The position of the particle was, however, some distance along the $x$-axis from its initial position. Note also that the path remains on one side of the $y$-axis and never crosses over. The magnetic intensity along a field line has minimum values on either side of the equator, and the path remains in the neighborhood of one of these minima. This single path suggests that a particle may only enter or leave the NA region through a small 'hole' on the velocity sphere.

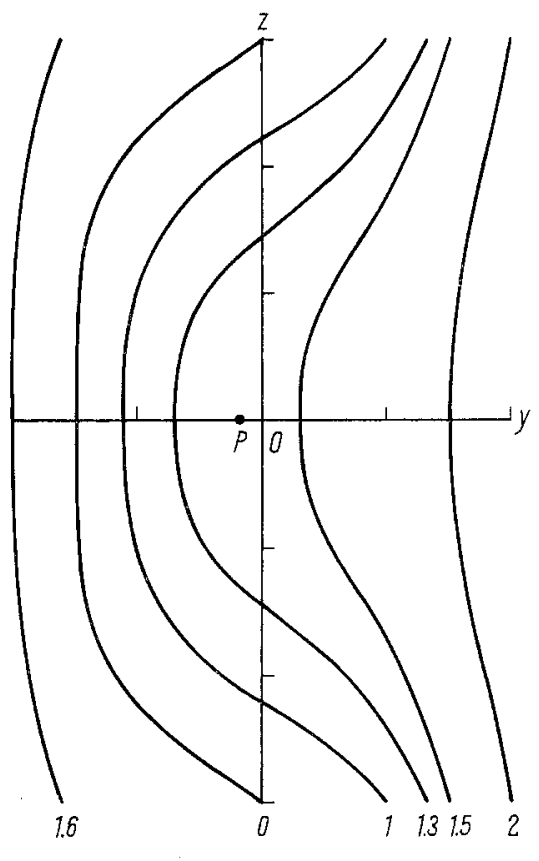

Figure 11

The magnetic field lines of a minimum $B$ configuration
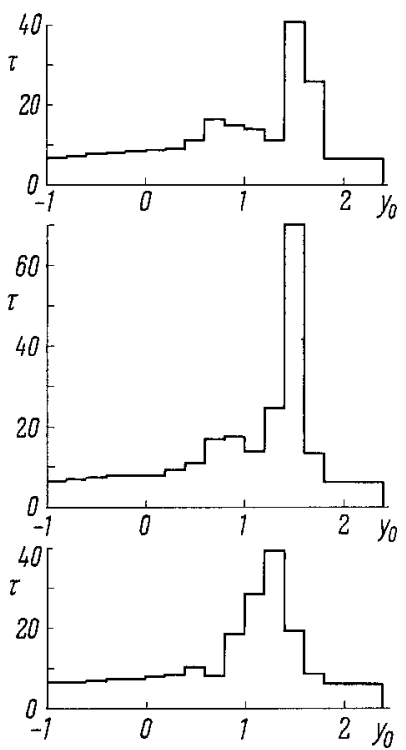

Figure 12

The time $\tau$ for a path to emerge from the non-adiabatic region as a function of $y_{0}$ for three different minimum $B$ fields
(a) $E=0.0$
(b) $E=0.2$
(c) $E=0.4$

\section{Conclusion}

Magnetic field configurations of the type shown in Figure 3 have been considered many times in astrophysical and geophysical contexts, and also in connection with the possibility of plasma containment in magnetic mirror devices. Some of the theories consider acceleration of the ambient electrons to suprathermal energies, and involve non-stationary processes outside the scope of the present work (GrovaNELII [13], Dungey $[14,15,16]$, Parker $[17,18]$ and Akasofu and Chapman [4]). We may, however, consider the relevance of our results to the theory of quiet auroral arcs, and comment upon the motion of charged particles in steady magnetic fields. 


\section{5 a Pitch angle scattering in the magnetosphere}

Quiet auroral arcs are produced by the entry into the earth's atmosphere of electrons that can penetrate to auroral levels. This requires that their equivalent equatorial pitch angles are only a few degrees. According to the theory of AKASOFU and CHAPMAN [4], a continuing supply of such electrons is provided by scattering during their passage through a non-adiabatic field region near the plane of the dipole equator. The model non-adiabatic field region here considered is an idealization of the supposed actual non-adiabatic field region associated with quiet auroral arcs.

Figure 14 illustrates the present discussion of scattering into orbits of small exit pitch angle in the non-adiabatic field region of Figure 3 . It shows a hemisphere in the velocity space, with a small central circular area EX representing the area of exit velocities of pitch angle up to $1^{\circ}$. The curve $C_{1}$ shows for the $z_{0}$ range $\mathrm{N}\left[0 \leq z_{0} \leq Z_{0}\right.$ $(=0.035)]$ the locus of the centres of the entry velocity areas corresponding to the exit area EX. For three values of $z_{0}$ (cf. Tables 1, 4 and 5) the shapes and orientations of the entry areas are shown, and also, to correct scale, the lengths of greatest extension of these areas; but the thickness (actually very small on this scale) is shown exaggerated; it can be inferred from the curve $A_{1}$ of Figure 15, which for this range of $z_{0}$ shows $\mathfrak{R}$, the ratio of the entry area to the area EX. The curves $C_{2}$ in Figure 14 and $A_{2}$ in Figure 15 similarly illustrate the entry areas corresponding to the exit area $\mathrm{EX}$, for $z_{0}$ range $\mathrm{F}\left[z_{0}>Z_{3}(=0.3)\right]$. It should be noted that in all cases the ratio to the entry area EX is never less than unity; but it is not large $(\sim 2$ at most). The curve $C_{3}$ shows the locus of the centres of the entry areas for the I range $Z_{1} \leq z_{0} \leq Z_{2}$.

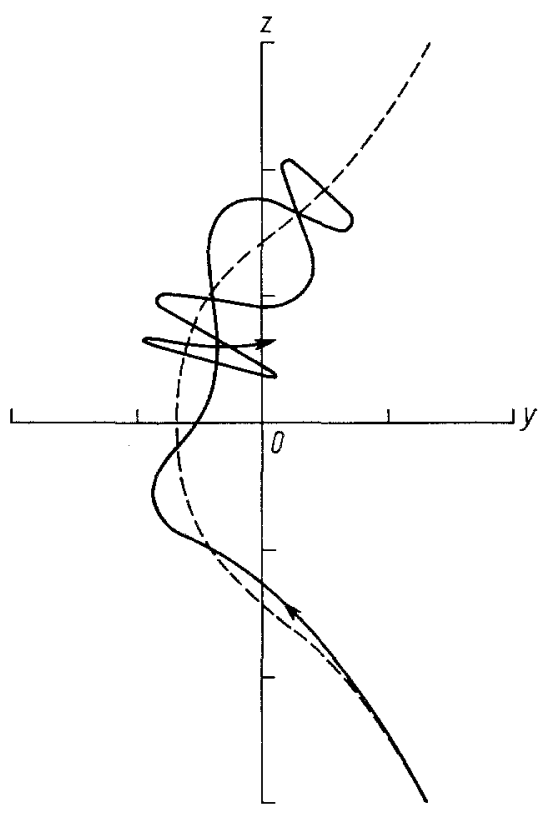

Figure 13

A highly complicated path which remained in the non-adiabatic region of Figure 11 for a long time 
The entry areas are long and thin. Their extent may be estimated from Tables 4 and 5 , and their shape visualized from Figure 10. (Note that the point on $C_{3}$ corresponding to $z_{0}=0.104$ is omitted from Figure 14 . It lies very near EX.)

Figure 15 shows by curves $A_{1}$ and $A_{2}$, for the respective $z_{0}$ ranges $\mathrm{N}$ and $\mathrm{F}$, how $\Re$ varies with $z_{0}$; note that the $z_{0}$ scales are different for the two curves. The curve $A_{1}$ with its $z_{0}$ scale increased tenfold (so that $z_{0}$ would range up to 0.4 ) would fit the variation of $z_{0}$ for the discontinuous field model of $\S 2$ remarkably well.

The direction of increasing $z_{0}$ in Figure 14 is shown on curves $C_{1}, C_{2}, C_{3}$ by arrows. Along $C_{1}$ the entry area and its polar angle increase with $z_{0}$, and its shape becomes more elongated along the azimuthal direction; along curve $C_{2}$ these changes are reversed, and the orbit becomes adiabatic beyond about $z_{0}=1$.

In the magnetosphere the occurrence of an equatorial non-adiabatic field region NA is the result not of local causes, but of development in the distribution of energetic particles over a great volume. Initially the equatorial pitch angle distribution of the particles in the field tubes passing through the region NA may be isotropic or not very far removed from isotropy. The particles with small equatorial pitch angles that are moving earthward will enter the atmosphere and create some auroral light. The particles moving towards the equator in the entry areas (or with the velocity directions in these areas) for different values of the magnetospheric parameter corresponding to $z_{0}$, will leave the NA region with small pitch angles corresponding to the area EX in Figure 14. These particles will pass onward into the polar atmosphere, and prolong the emission of auroral light. The other particles in these field tubes, which leave the region NA, will travel on towards mirror points above the atmosphere, and then return equatorwards with perhaps the same polar angle (in Figure 14) as before, but with a different azimuth. Part of their entry areas are likely to overlap the special entry areas shown for various values of $z_{0}$ in Figure 14 ; to the extent that

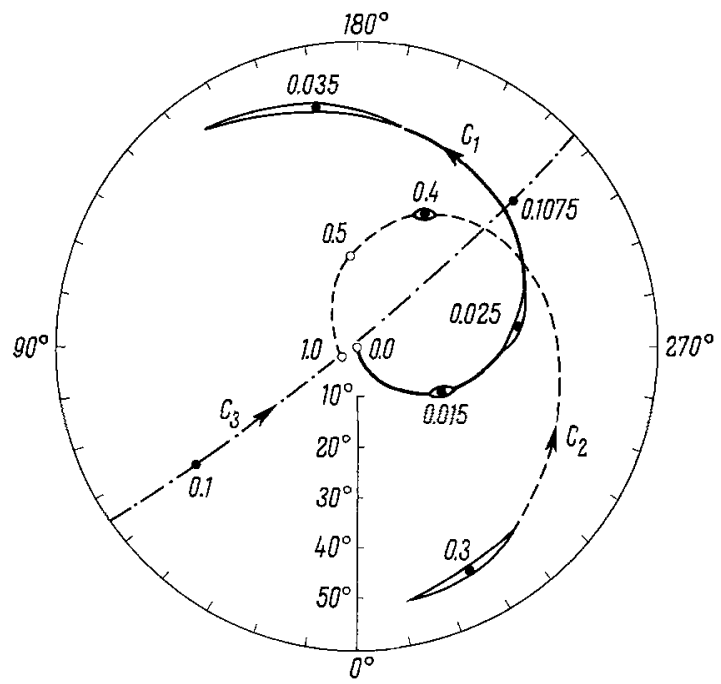

Figure 14

The locus on the unit sphere of the ends of the velocity vectors when the particle emerges from the non-adiabatic region of Figure 3. The region EX surrounds the centre of this diagram 
this happens, there will be a renewed supply of exit particles with small pitch angles able in their turn to contribute to the auroral light.

We estimate that pitch angle scattering of the type here considered could prolong the lifetime of a quiet auroral arc by a factor of order $(180 / \pi)^{2} \div 3600$ at most, namely from one or two seconds to one or two hours; such a duration of a quiet arc is exceptional. The decay of the supply would, of course, be exponential, for if $n$ denotes the total number of particles and $\tau$ denotes the time of passage across the system

$$
\frac{d n}{d t} \doteqdot-\left(\frac{\pi}{180}\right)^{2} \frac{n}{\tau}
$$

The calculations therefore indicate that a non-adiabatic region along a neutral line could contribute greatly to the number of auroral particles accessible to the atmospheric loss cone. Changes spread throughout the Van Allen belt region will slowly change the location of the NA region. A brief examination of the case when the magnetic field decreases nearly but not exactly to zero shows that scattering of pitch angles occurs then also.

\section{5 b Magnetic mirror devices}

In the present problem there is apparently a field line $z_{0} \doteqdot 0.104$ for which a particle injected with zero pitch angle on entry into the NA region leaves it with zero pitch angle. It follows that on this field line no path can enter the loss cone, and plasma loss through pitch angle scattering will be minimized. However, it is unlikely that ideal conditions could be obtained such as are assumed here.

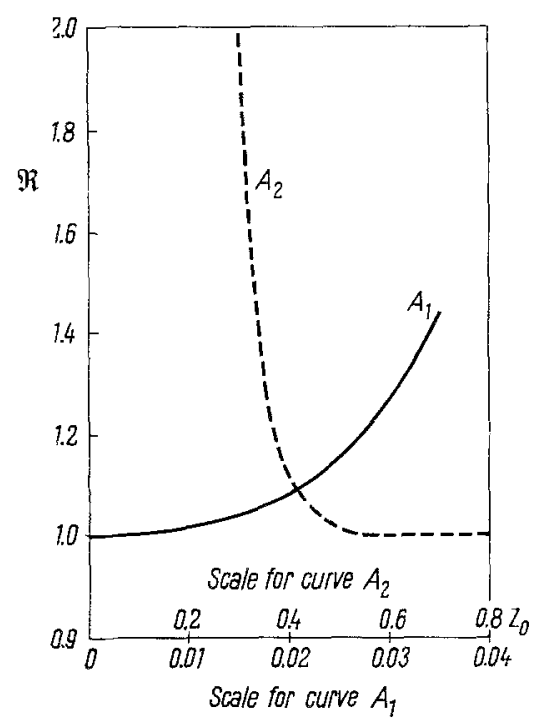

Figure 15

The ratio $\Re$ of the entry areas for Figure 14 to the exit area EX. The solid curve corresponds to the solid curve on Figure 14, and is referred to the lower scale of $z_{0}$. The broken curve corresponds to the broken curve in Figure 14 and is referred to the upper scale of $z_{0}$ 


\section{Acknoweledgements}

One of us (P. C. Kendall) thanks Professor S. F. Singer for his hospitality at the University of Maryland in April, 1961 and Bedford College, University of London, for leave of absence. We are grateful to Miss Astrik DEIRMENDJiAn and Mrs. RAYNoR GORDON for much help with the computations. The calculations were carried out chiefly at the University of London Computer Unit and the High Altitude Observatory, Boulder, Colorado.

\section{REFERENCES}

[1] H. ALFVÉN, Cosmical Electrodynamics, Oxford, ch. II (1950).

[2] S.-I. AkAsofu and S. Chapman, The ring curvent, geomagnetic disturbance and the Van Allen radiation belts, J. Geophys. Res. 66 (1961), 1321.

[3] S.-I. AKasofu, J.C. CaIN and S. Chapman, The magnetic field of a model radiation belt numerically computed, J. Geophys. Res. 66 (1961), 4013.

[4] S.-I. AkAsofu and S. Chapman, A neutral line discharge theory of the aurora polaris, Phil. Trans. Roy. Soc. London [A], 253 (1961), 359.

[5] T. Gold, Magnetic storms, Space Science Rev. 1 (1962), 100.

[6] E. N. PARKER, Dynamics of the geomagnetic storm, Space Science Rev. 1 (1962), 62.

[7] S. Chapman, Auroral phenomena, J. Phys. Soc. Japan 17 , Supp. A-II (1962), 619.

[8] S. CHAPMAN and P.C. KENDALI, The motion of charged particles in a model dicsontinuous magnetic field, Bul. Inst. Politeh. Iasi (Rumania), Vol. 8 (12), No. 3/4 (1962), 81.

[9] S. CHAPMAN, Scale times and scale lengths of variables, with geomagnetic and ionospheric illustrations, Proc. Phys, Soc. London 77 (1961), 424

[10] E. Åsröm, Electron orbits in hyperbolic magnetic fields, Tellus 8 (1956), 260.

[11] G. V. SHEFFIELD, An axially symmetric miniwn $B$ configumation, MATT-256, Plasma Physics Laboratory, Princeton University (1964).

[12] K. L. McDonald, Topology of steady current magnetic fields, Amer. J. Phys. 22 (1954) 586.

[13] R. G. GIovanelli, Magnetic and electric phenomena in the sun's atmosphere associated with sunspots, Monthly Notices, Royal Astron. Soc. 107 (1947), 338.

[14] J. W. Dungey, Conditions for the occurrence of electrical discharge in astrophysical systems, Phil. Mag. 44 (1953), 725.

[15] J. W. Dungey, Cosmic Electrodynamics, Cambridge (1958), 99.

[16] J. W. Dungey, The interplanetary field and auroral theory, J. Phys. Soc. Japan 17, Supp. A-II (1962), 15.

[17] E. N. PARKER, Sweet's mechanism for merging magnetic fields in conducting fluids, J. Geophys. Res. 62 (1957), 509.

[18] E. N. PARKER, The solar flare phenomenon and the theory of reconnexion and annihilation of magnetic fields, Astrophys. J., Supp. 77, 8 (1963), 177.

(Received 2nd September 1964) 